\title{
ON THE DIVERGENCE OF EXTENSION PROCEDURES IN ISOL THEORY
}

\author{
T. G. MCLAUGHLIN
}

\begin{abstract}
We show that the Myhill and Nerode extensions begin to disagree on the domain of the Nerode extension at a point in the arithmetical hierarchy $<\Delta_{3}^{0}$. This disagreement, at level $\Delta_{3}^{0}$, goes hand in hand with a certain way in which the Myhill extension fails, at $\Delta_{3}^{0}$, to commute with composition.
\end{abstract}

1. Introduction. In the theory of isols, there are two classical procedures for extending a numbertheoretic function $f\left(x_{1}, \ldots, x_{n}\right)$ to an isoltheoretic partial function $F\left(X_{1}, \ldots, X_{n}\right)$; "extension", of course, implies that $f \subseteq F$. The first of these procedures, historically, is the Myhill extension, lifting $f$ to $f_{M}$, which proceeds by way of application of the so-called normal combinatorial operators [1]. The other procedure is the Nerode extension [2], lifting $f$ to $f_{\Lambda}$, which uses what are called recursive frames. Each method has its special advantages: the Myhill technique extends each combinatorial function $f$ to a total isoltheoretic function $f_{M}$ (whereas $f_{\Lambda}$ is in general not defined for all $n$-tuples of isols, i.e., is "strictly partial"); on the other hand, the Nerode method is highly flexible in that it requires only partial operators (so-called "numerical frame maps") as opposed to the more rigidly circumscribed total operators called "combinatorial". It is well known (see [2]) that the two procedures give the same results when applied to the core class of $f$ 's studied by isol theorists, i.e., the class of recursive combinatorial functions. However, once one departs from the class of recursive functions, certain blemishes begin to appear (even though both procedures retain utility for the study of the isols); e.g., if $f(x)$ and $g(x)$ are combinatorial but $f$ is not recursive, then it need not be true that $f_{M}\left(g_{M}(X)\right)=(f \circ g)_{M}(X)$ holds for all isols $X$. Moreover, as already mentioned, if $f$ is nonrecursive then $\operatorname{dom}\left(f_{\Lambda}\right)$ will be a proper subset of the isols; indeed [2], $U \operatorname{dom}\left(f_{\Lambda}\right)$ will be a meager subset of $2^{N}$ relative to the usual (Cantor space) topology on $2^{N}$. Given this influx of "pathology" at higher-than-recursive levels, it should come as no surprise that there exist combinatorial functions $f: N \rightarrow N$ and isols $X$ such that $X \in \operatorname{dom}\left(f_{\Lambda}\right)$ and $f_{\Lambda}(X) \neq f_{M}(X)$. Indeed, one would expect this to be a well-known phenomenon, frequently alluded to in the literature. Yet I do not know of a single definite reference for it, and believe that it has been previously overlooked (though, in all likelihood, implicitly assumed): all previous effort seems to have been devoted to the very laudable enterprise of pushing the agreement between $f_{\Lambda}$ and $f_{M}$ as far "upward" as possible (see [3]). In

Received by the editors March 9, 1981.

1980 Mathematics Subject Classification. Primary 03D50.

(C) 1981 American Mathematical Society 0002-9939/81/0000-0571/\$02.25 
the present article, an example of disagreement between $f_{\Lambda}$ and $f_{M}$, on $\operatorname{dom}\left(f_{\Lambda}\right)$, is provided; my example exhibits a combinatorial function $f \in \Delta_{3}^{0}$ and, at the same time, shows the compositional misbehavior of the Myhill extension at level $\Delta_{3}^{0}$. (I am indebted to Erik Ellentuck for pointing out that my approach yields $f \in \Delta_{3}^{0}$ rather than merely $f \in \Delta_{4}^{0}$ as I had originally thought. In addition, Ellentuck pointed out to me that the proof actually turns on a particular realization of the pathological inequality $f_{M}\left(g_{M}(X)\right) \neq(f \circ g)_{M}(X)$.)

I shall assume familiarity on the reader's part with the principal contents of [1] and [2], as well as with the standard notations and terminologies of ordinary recursion theory.

2. "Bad" combinatorial functions in $\Delta_{3}^{0}$. Let $f: N \rightarrow N$ be a combinatorial function; i.e., $f(x)=\sum_{i=0}^{x} c_{\mathrm{i}}\left(\begin{array}{l}x \\ i\end{array}\right)$ holds with $c_{i}>0$ for all $i . f$ is said to be recursively bounded if there is a recursive function $g: N \rightarrow N$ such that $(\forall i)[f(i)<g(i)]$; equivalently, we can ask for a recursive $h$ such that $(\forall i)\left[c_{i}<h(i)\right]$. If the function $g$ (the function $h$ ) is merely recursive in $\mathbf{d}$, where $\mathbf{d}$ is some degree, we call $f$ d-bounded.

2.1. LemMa. There exists a recursively bounded combinatorial function $f \in \Delta_{3}^{0}-\Delta_{2}^{0}$ such that the function $f(2 x)$ is recursive.

Proof. Let $\left\langle\phi_{e}^{A} \mid e \in N\right\rangle$ be Kleene's $T^{A}$-predicate enumeration [5, §65] of the class of those unary partial numbertheoretic functions that are partial recursive in $A$, where $A$ is a fixed set (of numbers) of degree $\boldsymbol{\sigma}^{0}$. Then every unary $\Delta_{2}^{0}$ function is $=\phi_{e}^{A}$ for some $e$. Furthermore, if we let $F(e, x, y)$ be the predicate " $\phi_{e}^{A}(x)$ is defined and $=y$ " then $F(e, x, y)$ is of degree $\leqslant 0^{\prime \prime}$; hence, any function recursive in $F(e, x, y)$ is $\Delta_{3}^{0}$. We shall define $f$ by induction. The first couple of steps in the construction of $f$ will be given explicitly; it should then be clear to the reader what is going on in our formulation of the induction step.

Step 0. Set $f(0)=0$. If $\neg F(0,1,2)$, define $f(1)=2$; if $F(0,1,2)$, define $f(1)=1$. Observe that there are two possibilities for $\left\langle c_{0}, c_{1}\right\rangle$ : either $\left\langle c_{0}, c_{1}\right\rangle=\langle 0,1\rangle$ or $\left\langle c_{0}, c_{1}\right\rangle=\langle 0,2\rangle$. In the former case, $f(2)$ will have to be $\rangle 2$, while in the latter we must have $f(2) \geqslant 4$. To be sure that $f$ ends up combinatorial, therefore, we must play safe and define $f(2)$ to be at least 4 .

Step 1. Set $f(2)=4$. Then there are the following possibilities for $\left\langle c_{0}, c_{1}, c_{2}\right\rangle$ : $\langle 0,1,2\rangle,\langle 0,2,0\rangle$. This implies that for safety's sake $f(3)$ will have to be taken at least as large as $\max \left\{\left(\begin{array}{l}3 \\ 1\end{array}\right)+2\left(\begin{array}{l}3 \\ 2\end{array}\right), 2\left(\begin{array}{l}3 \\ 1\end{array}\right)\right\}=\max \{9,6\}=9$. If $\neg F(1,3,9)$, set $f(3)=9$; if $F(1,3,9)$, set $f(3)=10$. This gives rise to the following possibilities for $\left\langle c_{0}, c_{1}, c_{2}, c_{3}\right\rangle:\langle 0,1,2,0\rangle$ or $\langle 0,2,0,3\rangle$ if $f(3)=9 ;\langle 0,1,2,1\rangle$ or $\langle 0,2,0,4\rangle$ if $f(3)=10$. Therefore, $f(4)$ will have to be taken

$$
\begin{aligned}
& \geqslant \max \left\{\left(\begin{array}{l}
4 \\
1
\end{array}\right)+2\left(\begin{array}{l}
4 \\
2
\end{array}\right), 2\left(\begin{array}{l}
4 \\
1
\end{array}\right)+3\left(\begin{array}{l}
4 \\
3
\end{array}\right),\left(\begin{array}{l}
4 \\
1
\end{array}\right)+2\left(\begin{array}{l}
4 \\
2
\end{array}\right)+\left(\begin{array}{l}
4 \\
3
\end{array}\right), 2\left(\begin{array}{l}
4 \\
1
\end{array}\right)+4\left(\begin{array}{l}
4 \\
3
\end{array}\right)\right\} \\
& =\max \{16,20,20,24\}=24 .
\end{aligned}
$$

Step 2. Set $f(4)=24$. Calculate the resulting set of possibilities for $\left\langle c_{0}, c_{1}, c_{2}, c_{3}, c_{4}\right\rangle$, and then advance toward Step 3 along exactly the same lines as in the preceding two steps. 
For a precise description of the inductive passage from Step $\boldsymbol{n}$ to Step $\boldsymbol{n}+1$, we assume that Step $n$ has been concluded and that, with its conclusion, we have completed the definition of $f(x)$ for $x<2 n+1$. We assume, moreover, that along with the first $2 n+1$ values of $f$ we have defined sequences $\left\langle c_{0}, \ldots, c_{2 n+1}\right\rangle$ and $\left\langle k_{1}, k_{3}, \ldots, k_{2 n+1}\right\rangle$ of nonnegative integers, in such a way that the following conditions hold: (a) $f(x)=\sum c_{i}\left(\begin{array}{l}x \\ i\end{array}\right)$, for $x<2 n+1$; (b) for all $j<n$, $\neg F(j, 2 j+1, f(2 j+1))$; (c) for each $j \leqslant n$, either $f(2 j+1)=k_{2 j+1}$ or $f(2 j+1)$ $=k_{2 j+1}+1$; (d) the sequence $\left\langle k_{1}, k_{3}, \ldots, k_{2 n+1}\right\rangle$ has been computed effectively, i.e. with no appeals to oracles for information; (e) the values $f(2 j), j<n$, have all been computed effectively. Now, in order to have secured condition (b), we will have needed to appeal to an oracle for $F$ in dealing with odd arguments $<2 n+1$. The sequence $\left\langle c_{0}, \ldots, c_{2 n+1}\right\rangle$ has, therefore, been obtained with the aid of an oracle for $0^{\prime \prime}$. Nevertheless, conditions (c) and (e) provide a finite set $S=$ $\left\{\left\langle{ }^{i} c_{0}, \ldots,{ }^{i} c_{2 n+1}\right\rangle \mid i \in I\right\}$ of sequences of nonnegative integers, with the property that $\left\langle c_{0}, \ldots, c_{2 n+1}\right\rangle \in S$; moreover, in view of condition (d), the exact contents of the set $S$ can be effectively determined. We may therefore carry out Step $n+1$ as follows.

Step $n+1$. Set

$$
f(2 n+2)=\max \left\{\sum_{t=0}^{2 n+1}{ }^{i} c_{t}\left(\begin{array}{c}
2 n+2 \\
t
\end{array}\right) \mid\left\langle{ }^{i} c_{0}, \ldots,{ }^{i} c_{2 n+1}\right\rangle \in S\right\} .
$$

This gives rise to a well-determined set $S^{\prime}$ of possibilities for $\left\langle c_{0}, \ldots, c_{2 n+2}\right\rangle$, one such possibility for each sequence $\left\langle{ }^{i} c_{0}, \ldots,{ }^{i} c_{2 n+1}\right\rangle \in S$. Next, set $k_{2 n+3}=$ $\max \left\{\sum_{t=0}^{2 n+2} d_{t}\left({ }^{2 n+3} t\right) \mid\left\langle d_{0}, \ldots, d_{2 n+2}\right\rangle \in S^{\prime}\right\}$. If $\neg F\left(n+1,2 n+3, k_{2 n+3}\right)$, set $f(2 n+3)=k_{2 n+3}$; otherwise set $f(2 n+3)=k_{2 n+3}+1$. Finally, let $c_{2 n+2}$ and $c_{2 n+3}$ be nonnegative integers such that $f(x)=\Sigma c_{i}\left(\begin{array}{l}x \\ i\end{array}\right)$ holds for $x<2 n+3$. It is easily seen that conditions (a)-(e) remain satisfied with $n+1$ in place of $n$, and the induction step is complete.

It is evident that $k_{2 x+1}$ is a recursive function of $x$, that $\operatorname{deg}(f)<0^{\prime \prime}$ (i.e., $\left.f \in \Delta_{3}^{9}\right)$, and that $f(2 x)$ is recursive. $f$ is combinatorial by condition (a), since $c_{i}>0$ for all $i$. The fact that $f$ is not $\Delta_{2}^{0}$ is a consequence of condition (b) holding at the end of each step of the construction; while the recursively bounded character of $f$ follows from condition (c), in view of the recursiveness of the functions $k_{2 x+1}$ and $f(2 x)$. The proof of the lemma is complete.

2.2. Lemma. Let $f: N \rightarrow N$ be a $\mathbf{0}$-bounded combinatorial function such that $f \notin \Delta_{2}^{0}$, let $A$ be an infinite cosimple isol, and suppose that there exist nonnegative integers $m$ and $n$, with $n \geqslant 2$, such that the function $f(n x+m)$ is recursive. Then $f_{\Lambda}(n A+m)$ is defined and $f_{\Lambda}(n A+m) \neq f_{M}(n A+m)$. Moreover, if $g(x)=$ $f(n x+m)$ for all $x$ then $g_{M}(A) \neq f_{M}(n A+m)$.

Proof. Let $n x+m, n>1$, be such that $f(n x+m)$ is a recursive combinatorial function. Let $\Phi$ be the normal recursive combinatorial operator inducing $f(n x+m)$; and let $\alpha$ be an infinite, isolated, $\Pi_{1}^{0}$ set. If $A$ is the isol represented by $\alpha$, then $n A+m$ is represented by the set $\alpha^{\prime}=\{x \mid x<m\} \cup\{m+x \mid x \equiv 0$ $(\bmod n) \& x / n \in \alpha\} \cup \cdots \cup\{m+x \mid x \equiv n-1(\bmod n) \&(x-n+1) / n \in \alpha\}$. 
Let $\mathscr{F}=\{\langle\{x \mid x<m\} \cup\{m+x \mid x \equiv 0(\bmod n) \& x / n \in \beta\} \cup \cdots \cup\{m+x \mid x$ $\equiv n-1(\bmod n) \&(x-n+1) / n \in \beta\}, \Phi(\beta)\rangle \mid \beta$ is a finite subset of $N\}$. It is easy to see that $\mathscr{F}$ is a recursive $f$-frame. (Recall that $\Phi$ induces $f(n x+m)$, and that combinatorial operators are multiplicative.) Furthermore, it is clear, since combinatorial operators are monotone and satisfy the condition

$$
C(\alpha)=\cup\{C(\beta) \mid \beta \subseteq \alpha \& \beta \text { is finite }\},
$$

that the pair $\left\langle\alpha^{\prime}, \Phi(\alpha)\right\rangle$ is attainable from $\mathscr{F}$. Thus $f_{\Lambda}(n A+m)$ is defined and $=B$, where $B$ is the isol containing $\Phi(\alpha)$. Also, we have $B=g_{M}(A)$; so, to complete the proof of the lemma it suffices to show that $f_{M}(n A+m) \neq B$. Let $\Psi$ be the normal combinatorial operator inducing $f$. Then $\Psi\left(\alpha^{\prime}\right)$ is a representative of $f_{M}(n A+m)$; and, if $f_{M}(n A+m)=B$ holds then there must exist a one-to-one partial recursive function $p$ such that $\Phi(\alpha) \subseteq \operatorname{dom}(p) \& p(\Phi(\alpha))=\Psi\left(\alpha^{\prime}\right)$. Assume, for an argument by contradiction, that such a function $p$ exists. Now, $\Psi\left(\alpha^{\prime}\right)=$ $\left\{j(x, y) \mid D_{x} \subseteq \alpha^{\prime} \& y<c_{\operatorname{card}\left(D_{x}\right)}\right\}$; here $\left\langle D_{n} \mid n \in N\right\rangle$ is the usual "canonical" indexing of the finite sets, and $j: N^{2} \rightarrow N$ is the usual one-to-one effective pairing map from $N^{2}$ onto $N$. By (the proof of) Proposition 11 of [6, $\Phi(\alpha)$ is a $\Pi_{1}^{0}$ set. Hence $p(\Phi(\alpha))$, i.e., $\Psi\left(\alpha^{\prime}\right)$, is the difference of two $\Sigma_{1}^{0}$ sets. Certainly, then, $\Psi\left(\alpha^{\prime}\right)$ is of degree $\leqslant 0^{\prime}$. Let $h: N \rightarrow N$ be a function of degree $<0^{\prime}$ such that $(\forall i)\left[c_{i}<h(i)\right]$. We now specify a procedure for calculating the sequence $\left\langle c_{i} \mid i \in N\right\rangle$ recursively in $\boldsymbol{O}^{\prime}$; this will provide the desired contradiction, since it implies that $f \in \Delta_{2}^{0}$. To compute $c_{n}, n>0$, let $w$ be a number such that $D_{w}$ is an $n$-element subset of $\alpha^{\prime}$. Such a number $w$ can obviously be found through appeal to an oracle for $\boldsymbol{\sigma}$, by a procedure uniform in $n$. Next, find the set of all numbers $j(w, y)$ such that (i) $p^{-1}(j(w, y))$ is defined and belongs to $\Phi(\alpha)$ and (ii) $y<h(n)$. Clearly, this set can be exactly determined through appeal to an oracle for $\boldsymbol{\sigma}^{\prime}$, using a procedure uniform in $n$; moreover, its cardinality is $c_{n}$. Thu, $\operatorname{deg}(f)<0^{\prime}$; i.e., $f \in \Delta_{2}^{0}$. But $f \in \Delta_{2}^{0}$ is a contradiction, and the lemma is proved.

2.3. THEOREM. There exist a unary combinatorial function $f \in \Delta_{3}^{0}$ and a cosimple regressive isol $A$ such that (1) $A \notin \operatorname{dom}\left(f_{\Lambda}\right)$, (2) $2 A \in \operatorname{dom}\left(f_{\Lambda}\right)$, (3) $f_{\Lambda}(2 A) \neq$ $f_{M}(2 A)$, and (4) $g_{M}(A) \neq f_{M}(2 A)$ where $g(x)=f(2 x)$ for all $x$.

Proof. Let $f$ be as in Lemma 2.1; then, since each recursively bounded function is a fortiori $0^{\prime}$-bounded, $f$ meets the conditions set forth in the hypotheses of Lemma 2.2 with $n=2$ and $m=0$. Since $f$ itself is not recursive, it follows from results in [4] that if $A$ is a strongly universal, cosimple, regressive isol then $A \notin \operatorname{dom}\left(f_{\Lambda}\right)$. Lemma 2.2 provides the remaining three assertions of the theorem.

3. Some remarks about $\Delta_{2}^{0}$. Having found the example of $\$ 2$, it is natural to try to find a better example, that is, one in which $\operatorname{deg}(f)<\boldsymbol{0}^{\prime}$. It is known from results in [3] that any such $f$ would have to lie outside the class of " $R \uparrow$ " combinatorial functions. If we attempt to use the approach of $\$ 2$, of course mixing in some approximations, in order to obtain Theorem 2.3 with " $f \in \Delta_{3}^{0 \text { "' replaced by " } f \in \Delta_{2}^{0}}$ - $R \uparrow$ ", then Lemma 2.1 presents no difficuity: it is easy to construct a recursively bounded combinatorial function $f$ such that $f \in \Delta_{2}^{0}-R \uparrow$ and $f(2 x)$ is recursive. 
Lemma 2.2, however, presents a problem: in adapting its proof, we would attempt to produce a contradiction by arguing that if $f_{M}(n A+m)=B$ then $f$ is $R \uparrow$. The definition of $R \uparrow$, however, requires that in making successive approximations to an $n$-element subset $D_{w}$ of $\alpha^{\prime}$ we avoid ever making an overestimate of

$$
\operatorname{card}\left(\left\{j(w, y) \mid y<h(n) \& j(w, y) \in \operatorname{dom}\left(p^{-1}\right) \& p^{-1}(j(w, y)) \in \Phi(\alpha)\right\}\right) .
$$

We have so far found no way to avoid such temporary overestimates. It seems plausible, nonetheless, that an example can be located in $\Delta_{2}^{0}$, perhaps by a completely different line of argument but almost certainly by an argument involving some use of "priorities".

One might be tempted to go so far as to conjecture that any combinatorial function $f \in \Delta_{2}^{0}-R \uparrow$ is "bad", especially after noting, in connection with the framework of [3], that it is exactly the $R \uparrow$ combinatorial functions whose associated normal operators are "partial recursive". That conjecture, however, is false for a curiously "vacuous" reason: it is easy to see that if $f_{\Lambda}$ has any infinite isols in its domain then $f$ has an infinite partial recursive subfunction, and it is not difficult to construct a combinatorial function $f \in \Delta_{2}^{0}-R \uparrow$ such that $f$ has no infinite partial recursive subfunctions. Thus, we have at present little idea as to the exact contents of the class $\left\{f \mid f\right.$ is a unary combinatorial function in $\Delta_{2}^{0}$ and $f_{\Lambda}=f_{M}$ on $\left.\operatorname{dom}\left(f_{\Lambda}\right)\right\}$.

Since this paper was submitted, the more clear-cut of the problems posed or suggested in $\$ 3$ have been solved by Ellentuck. In particular, Ellentuck has found a refinement of the last part of the proof of Lemma 2.2, from which refinement it follows (via, say, the existence of minimal pairs of r.e. degrees) that the combinatorial function of Theorem 2.3 can be located in $\Delta_{2}^{0}$. He has, moreover, exploited the technique of "generic isols" to obtain very strong results in the noncosimple case. Ellentuck's work also implicitly contains the solution to the characterization problem for $\left\{f \in \Delta_{2}^{0}-R \uparrow \mid f\right.$ is combinatorial and $\left.f_{\Lambda} \subseteq f_{M}\right\}$ : it turns out to be just the set of $\Delta_{2}^{0}-R \uparrow$ combinatorial functions having no infinite partial recursive subfunctions!

\section{REFERENCES}

1. J. Myhill, Recursive equivalence types and combinatorial functions, Bull. Amer. Math. Soc. 64 (1958), 373-376.

2. A. Nerode, Extensions to isols, Ann. of Math. 73 (1961), 362-403.

3. E. Ellentuck, The positive properties of isolic integers, J. Symbolic Logic 37 (1972), 114-132.

4. On the degrees of universal regressive isols, Math. Scand. 32 (1973), 145-164.

5. S. C. Kleene, Introduction to metamathematics, Van Nostrand, Princeton, N. J., 1952.

6. J. C. E. Dekker, Infinite series of isols, Recursive Function Theory, Proc. Sympos. Pure Math., vol. 5, Amer. Math. Soc., Providence, R. I., 1962, pp 77-96.

Department of Mathematics, Texas Tech University, Lubbock, Texas 79409 